\title{
The utility of molecular diagnostics in tumour surveillance in Beckwith-Wiedemann syndrome - clinical genetics point of view in light of a case report
}

\author{
Justyna Drankowska1, Michał Kos , Andrzej Kościuk1, Joanna Nurzyńska-Flak² \\ 'Student Research Group in the Department of Paediatric Haematology, Oncology, and Transplantology, \\ Medical University of Lublin, Lublin, Poland \\ 2Department of Paediatric Haematology, Oncology, and Transplantology, Medical University of Lublin, Lublin, Poland
}

\section{ABSTRACT}

Beckwith-Wiedemann syndrome (BWS) is the most frequent congenital overgrowth syndrome. Its clinical features are variable, including macrosomia, hemihyperplasia, and macroglossia. The most important feature appears to be the increased risk of embryonal tumours, depending on the molecular aetiology of BWS. The underlying molecular abnormalities might be genetic or epigenetic, and they involve the segment of the genome - chromosome $11 \mathrm{p} 15$.

The aim of the study is to present the case report of a patient with BWS, who was at the highest molecular risk of developing embryonal tumour associated with this syndrome. The authors emphasise the importance of therapy individualisation in BWS due to variable cancer predispositions associated with molecular aetiology.

\section{KEY WORDS:}

malignant neoplasm, molecular diagnostics, Beckwith-Wiedemann syndrome, epigenetic defects.

\section{INTRODUCTION}

Beckwith-Wiedemann syndrome (BWS) is the most common among the genetic overgrowth syndromes [1]. Its prevalence is estimated at one in every 13,700 live births, equally in males and females $[2,3]$. The incidence appears to be higher in children after in vitro fertilisation [4].

The syndrome is named after Beckwith and Wiedemann, two doctors who described it with three cardinal features, i.e. exomphalos, macroglossia, and gigantism $[2,5]$. Since then, the associated phenotype has been expanded. Characteristic features of BWS include macrosomia, macroglossia, hemihyperplasia, omphalocele, visceromegaly, facial naevus flammeus, earlobe creases or pits, and neonatal hypoglycaemia [5-8]. What is most significant, BWS is associated with an increased risk of tumours in childhood. However, clinical manifestations are variable - affected individuals might have a number of features, whereas others might have only one or two. Clinical diagnostic criteria for BWS have not been established, but Shuman et al. published criteria in GeneReviews [9]: According to them, BWS should be considered when three major or one major and two minor criteria are present (Table 1) [9].

All these manifestations are caused by genetic and epigenetic abnormalities involving the imprinted band of the genome - chromosome 11p15. The process of imprinting is based on different expression of genes, depending on the parent who contributed them $[3,5,9,10]$. Imprinting control regions (ICR1 and ICR2), which are present in the band, such as $11 \mathrm{p} 15$, are responsible for regulation of the imprinted gene expression [2, 3, 5, 9-12] (Fig. 1).

\section{ADDRESS FOR CORRESPONDENCE:}

Justyna Drankowska, Department of Paediatric Haematology, Oncology, and Transplantology, Medical

University of Lublin, 6 Prof. A. Gębali St., 20-093 Lublin, Poland, e-mail: justyna.drankowska@gmail.com 
TABLE 1. Major and minor findings associated with Beckwith-Wiedemann syndrome (BWS)

\section{Major findings}

Positive family history (one or more family members with a clinical diagnosis of BWS or a history or features suggestive of BWS)

Macrosomia (traditionally defined as weight and length/height $>97^{\text {th }}$ centile)

Macroglossia (large size of the tongue)

Hemihyperplasia (asymmetric overgrowth of one or more regions of the body)

Visceromegaly involving one or more intra-abdominal organs including liver, spleen, kidneys, adrenal gland or pancreas

Omphalocele (also called exomphalos) or umbilical hernia

Cytomegaly of the fetal adrenal cortex (pathognomonic)

Embryonal tumour (e.g., Wilms tumour, hepatoblastoma, neuroblastoma - especially patients with CDKN1 mutations [13], rhabdomyosarcoma) in childhood

Renal abnormalities including structural abnormalities, nephromegaly, nephrocalcinosis or later development of medullary sponge kidney

Anterior linear ear lobe creases and/or posterior helical ear pits

Placental mesenchymal dysplasia

Cleft palate (rare in BWS)

Cardiomyopathy (rare in BWS)

Minor findings associated with BWS

Pregnancy-related findings including polyhydramnios and prematurity

Neonatal hypoglycaemia

Vascular malformations such as facial nevus flammeus

Characteristic facies including midface hypoplasia and infraorbital creases

Structural cardiac anomalies or cardiomegaly

Diastasis recti

Advanced bone age (common in overgrowth/endocrine disorders)

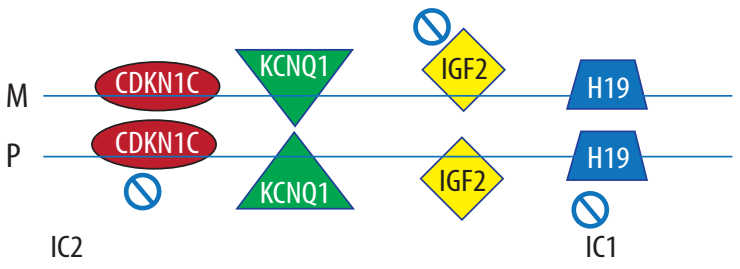

FIGURE 1. Schematic representation of the imprinting control regions (IC) on chromosome 11p15. IC1 contains imprinted embryonic growth factor IGF2 (expressed from paternal allele) and the noncoding RNA H19 (expressed from maternal allele). Whereas, IC2 contains the cyclin-dependent kinase (CDK)-inhibitor 1C (CDKN1c) protein (expressed from the maternal allele) and the noncoding RNA (KCNQ1); M - maternal allele, $\mathrm{P}$ - paternal allele

The majority of the known cases of BWS are caused by epigenetic abnormalities such as methylation or modification of histones $[2,9]$. Epigenetic defects only change gene expression without any impact on the DNA sequence. The most frequent cause of BWS (50\% of cases) is loss of methylation (LOM) in the maternal ICR2, which results in reduced expression of the cyclin-dependent kinase inhibitor $1 \mathrm{C}$ gene (CDKN1C). However, this pattern is not the only one observed in BWS. The complex regulation might also be disrupted by other mechanisms, for instance the gain of methylation at IC1 on the maternal chromosome $[1,2]$. In addition to epimutations in $11 \mathrm{p} 15$, mutations in CDKN1C and chromosome abnormalities such as paternal uniparental isodisomy of $11 \mathrm{p} 15.5$ (upd[11]pat) can also be related to BWS [1, 2, 5, 12].

The syndrome is definitively diagnosed by a combination of assessment of clinical features and genetic tests. Methylation-sensitive multiplex ligation probe analysis (MS-MLPA) is a widely used diagnostic tool for detection of DNA methylation changes and copy number changes [3]. However, the diagnosis in many children is only clinical due to the limited sensitivity of genetic tests [2].

\section{CASE REPORT}

A four-month-old boy was referred to our clinic for genetic evaluation because of macroglossia and left-sided hemihyperplasia. He was born at 40 weeks to a 21-yearold primigravida by vaginal delivery. He weighed $4.160 \mathrm{~kg}$ and was $53.5 \mathrm{~cm}$ in length. Both weight and length were above the $95^{\text {th }}$ centile. He had an Apgar score of 9. Initially, the boy was breastfed. However, macroglossia hindered breastfeeding as the only method of nutrition and resulted in partial artificial feeding. In terms of neonatal glycaemia, the data were normal. The family history was not contributory. Dysmorphology examination revealed 
also capillary malformation on the abdominal skin and loud murmur due to patent ductus arteriosus. The clinical findings (two major findings: macroglossia, hemihyperplasia, minor findings: structural cardiac anomaly [mitral valve incompetence and patent ductus arteriosus]) aroused suspicion of BWS, which was communicated to the parents. Therefore, genetic tests were performed on a sample of blood. Chromosome analysis reported normal 46, XY. However, epigenetic abnormalities were detected: DNA methylation test of $11 \mathrm{p} 15$ revealed gain of methylation of ICR1, which established the BWS diagnosis (Fig. 2).

As a result, tumour surveillance was immediately commenced, consisting of abdominal ultrasonography and serum alpha-fetoprotein assessment every three months until eight years of age. Serum alpha-fetoprotein, blood glucose, electrolytes, urinary creatinine, and complete blood count remained within reference levels. Nevertheless, a routine screening ultrasound revealed a lesion in the left kidney at the age of seven months. After one month of observation, enlargement of the lesion was observed. A subsequent CT scan supported a probable diagnosis of a Wilms tumour (WT), without detecting any distant metastases (Fig. 3). The biopsy confirmed blastemal predominant nephroblastoma.

The patient was treated according to the SIOP (International Society of Paediatric Oncology) Wilms tumour 93-01 protocol [14]. He received neoadjuvant chemotherapy consisting of vincristine and actinomycin for four weeks. After evaluation with post-treatment CT, left partial nephrectomy was performed. Local tumour stage according to SIOP was I [14]. The patient completed postoperative chemotherapy and did not require radiotherapy. He is currently in remission 10 years after initial diagnosis and attends regular school. The neurological development of the patient is normal. As the limb length discrepancy decreased, no surgery was needed to equalise leg length. The patient currently wears orthopaedic shoe insoles. What is more, the size of the tongue decreased.

\section{DISCUSSION}

The case depicts the most important complications of BWS and shows how they evolve consecutively during the first years of life. Of particular importance is the fact that children with BWS are at increased incidence risk for developing embryonal tumours such as WT, hepatoblastoma, neuroblastoma, or rhabdomyosarcoma $[5,15]$. The risk for these tumours is approximately $7.5 \%$ until eight years of age, and then it gradually decreases to very close to the general population risk $[2,6,9]$. The susceptibility to tumours varies considerably depending on themolecular aetiology of BWS. Another clinically significant fact is that tumour types differ among molecular subgroups $[11,16]$.

The highest risk for tumour (approximately 28\%) is associated with the gain of methylation (GOM) at IC1, which

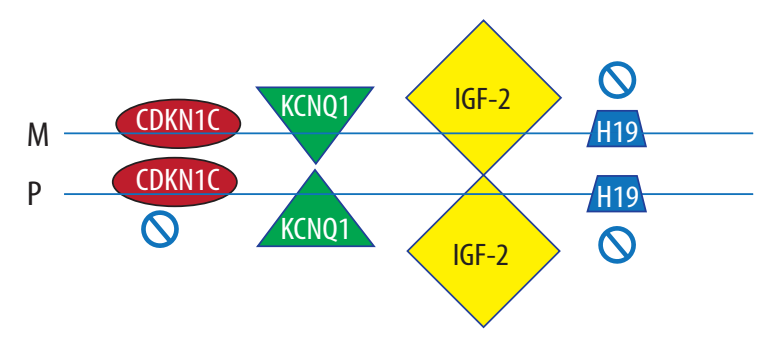

FIGURE 2. The patient's genetic pattern showing biallelic expression of IGF-2 and loss of $\mathrm{H} 19$ expression due to the gain of methylation in the maternal IC1

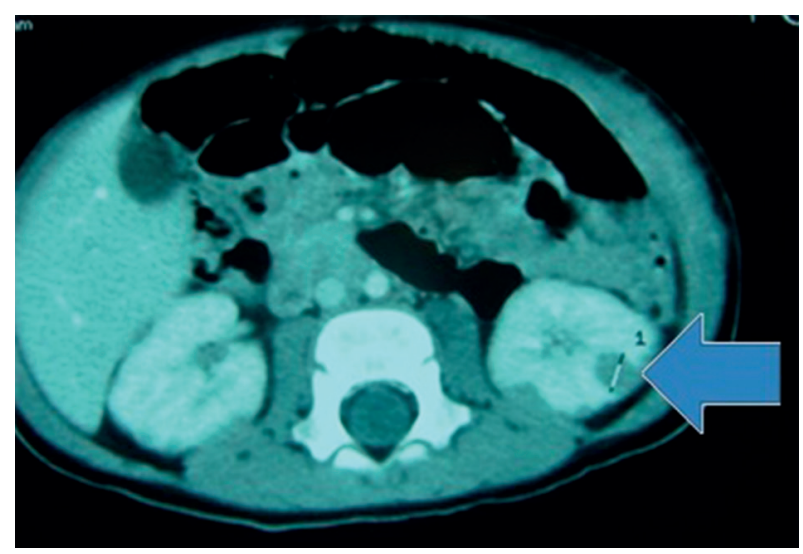

FIGURE 3. CT scan showing a focal non-enhancing hypodense lesion in the left kidney (arrow)

occurred in our patient. Such an abnormality is relatively infrequent and constitutes only $5 \%$ of all known BWS causes [9]. Gain of methylation at the ICR1 gene leads to biallelic expression of insulin-like growth factor-2 (IGF-2) and loss of expression of H19 mRNA. The excessive expression of IGF-2, the hormone promoting growth and proliferation of cells in many different tissues, leads to organomegaly and further tumour development [1, 2]. Moreover, H19 mRNA might act as a tumour suppressor [3].

Although patients with IC1 GOM have the highest tumour risk among all molecular subgroups of patients with BWS, they are mostly predisposed to developing WT (95\% malignant tumours in this group). Thus, screening in BWS patients with IC1 hypermethylation should only be aimed at early WT detection $[11,16]$. As far as tumour risk in BWS patients with upd(11)pat is concerned, it is estimated to be as high as $16 \%$. However, patients with this abnormality are predisposed to develop any of the tumour types seen in BWS, including WT (7.9\%), hepatoblastoma (3.5\%), neuroblastoma (1.4\%), and adrenal tumours $(1.1 \%)$. In terms of CDKN1C mutation, the occurrence of this mutation in a patient should be associated with increased predisposition to neuroblastoma (4.2\%) [16]. Urinary catecholamine metabolites such as vanillylmandelic acid (VMA) and homovanillic acid (HVA) are well-known markers that could be increased in this neoplasm [17]. Conversely, patients with IC2 LOM seem to have the most favourable prognosis because of the lower tumour incidence (overall risk - 2.6\%) compared to other molecular subgroups. As a result, tumour 


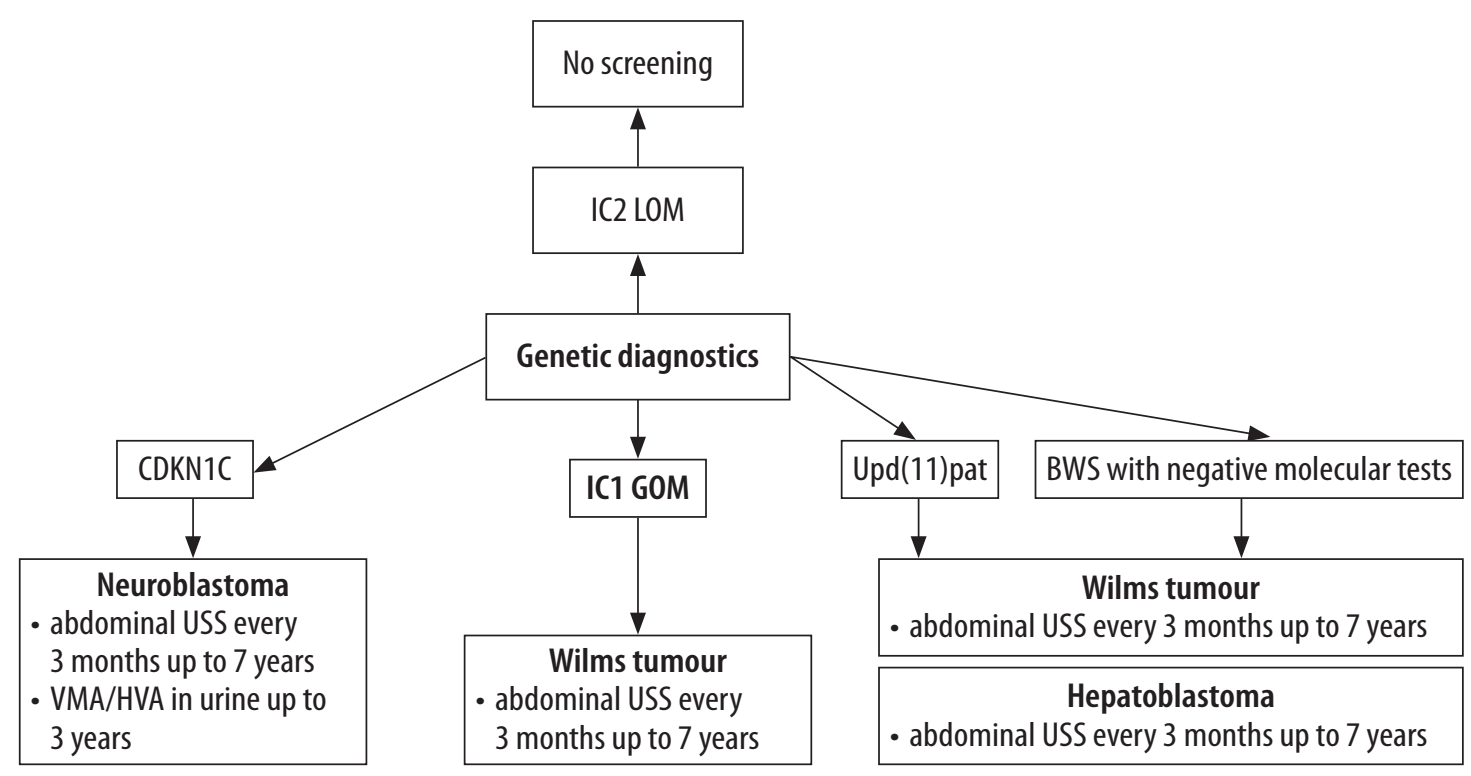

FIGURE 4. Suggested tumour surveillance in patients with Beckwith-Wiedemann syndrome depending on molecular aetiology

screening should be individualised depending on the molecular subgroup. Abdominal ultrasound scan (USS) is recommended in every molecular subgroup except for IC2 LOM [16] (Fig. 4).

As far as our patient is concerned, he developed the most frequent tumour (60\% of all tumours) observed in children with BWS, i.e. nephroblastoma (WT) [12]. According to Maas et al., the median age at which BWS patients develop WT is 24 months [11]. The most favourable outcome appears to be in children younger than 24 months with localised disease, as in our patient [18]. Nevertheless, blastemal predominant WT reported in our patient relates to worse prognosis in comparison with other subtypes [19]. Due to the highest risk for WT associated with this syndrome, the patient underwent abdominal ultrasound examinations every three months until the age of eight years. In order to detect hepatoblastoma, alpha-fetoprotein (AFP) levels were measured at intervals ranging from six weeks to three months until the age of four years [13]. Nevertheless, in 2016 Maas et al. showed that hepatoblastoma never occurred in patients with IC1 GOM [11]. It remains in accordance with the recent Consensus Statement, which does not recommend AFP screening in any molecular subgroup. It is associated with difficulties in interpreting serum AFP levels as well as the burden of repeated blood sampling [16].

The purpose of screening revolves around detecting tumours at a less advanced stage. Tumours detected earlier are associated with less extensive surgery and less intensive chemo- and radiotherapy, thus with better survival [12]. Benefitting from regular tumour surveillance initiated in our patient after clinical and molecular diagnosis, WT was detected at an early stage, being amenable to nephron-sparing surgery [20]. The fact that WT has the potential for both local spread and distant metastases makes early detection important [21].
Apart from that, clinical manifestations of this syndrome are variable, and they seem to depend on molecular type. Our patient exhibited clinical features, i.e. macroglossia and limb length discrepancy, which appear to be common in a group of patients with IC1 GOM in the study by Maas [11]. Conversely, omphalocele seems to be a real rarity for this molecular group. Neither our patient nor 20 patients with IC1 GOM in the above-mentioned study by Maas suffered from this condition [11]. What is important, neonates born with an omphalocele ought to be monitored for hypoglycaemia [22]. Hypoglycaemia in BWS occurs in approximately $50 \%$ of children with BWS and can be explained by hyperinsulinism due to the hypertrophy and hyperplasia of the islets of Langerhans [23]. Although the majority of cases are transient and can be treated by frequent feedings, in up to $20 \%$ of neonates hypoglycaemia might be persistent and require medications such as diazoxide and octreotide or even partial pancreatectomy in the most severe cases $[16,22]$.

It is extremely significant that the clinical manifestations in our patient have become less noticeable with age, as in the literature. Macroglossia has a tendency for regression to the size of the oral cavity [24]. Nevertheless, in some patients tongue reduction and speech therapy are needed. As far as limb length discrepancy is concerned, it is likely to become less distinct. In some cases an evaluation and follow-up by orthopedists should be performed in order to provide information about surgery involving epiphysiodesis [2].

\section{CONCLUSIONS}

The presented case demonstrates importance of screening strategies aimed at patients who are at high risk for cancer due to BWS. First of all, proper dysmorphology examination is vital to establish the diagnosis of 
BWS, which is clinical in many children. Secondly, the treatment of patients with BWS should be individualised depending on molecular aetiology. Above all, the most important issue is the multidisciplinary approach to these patients based on the cooperation of various specialists including paediatric oncologists, orthopaedists, otolaryngologists, surgeons, orthodontists, speech pathologists, etc. Such appropriate treatment might help in obtaining long-term positive results.

\section{DISCLOSURE}

The authors declare no conflict of interest.

\section{REFERENCES}

1. Mussa A, Molinatto C, Baldassarre G, et al. Cancer Risk in BeckwithWiedemann Syndrome: A Systematic Review and Meta-Analysis Outlining a Novel (Epi)Genotype Specific Histotype Targeted Screening Protocol. J Pediatr 2016; 176: 142-149.e1.

2. Pappas JG. The Clinical Course of an Overgrowth Syndrome, From Diagnosis in Infancy Through Adulthood: The Case of Beckwith-Wiedemann Syndrome. Curr Probl Pediatr Adolesc Health Care 2015; 45: 112-117.

3. Giżewska M, Wilk M, Patalan M, et al. The significance of molecular studies in the long-term follow-up of children with Beckwith-Wiedemann syndrome. Turk J Pediatr 2014; 56: 177-182.

4. Ferianec V, Bartova M. Beckwith-Wiedemann syndrome with overlapping Perlman syndrome manifestation. J Matern Neonatal Med 2014; 27: 1607-1609.

5. Cohen MM. Beckwith-Wiedemann Syndrome: Historical, Clinicopathological, and Etiopathogenetic Perspectives. Pediatr Dev Pathol 2005; 8: 287-304.

6. Brzezinski J, Shuman C, Choufani S, et al. Wilms tumour in BeckwithWiedemann Syndrome and loss of methylation at imprinting centre 2: revisiting tumour surveillance guidelines. Eur J Hum Genet 2017; 25: 1031-1039.

7. Brioude F, Lacoste A, Netchine I, et al. Beckwith-Wiedemann Syndrome: Growth Pattern and Tumor Risk according to Molecular Mechanism, and Guidelines for Tumor Surveillance. Horm Res Paediatr 2013; 80: 457-465.

8. Style CC, Cruz SM, Lau PE, et al. Surgical Outcomes of Patients with Beckwith-Wiedemann Syndrome. J Pediatr Surg 2018; 53: 1042-1045.

9. Shuman C, Beckwith JB, Weksberg R. Beckwith-Wiedemann Syndrome. In: Adam MP, Ardinger HH, Pagon RA, et al. (eds.). GeneRev [Internet], University of Washington, Seattle 1993-2018.

10. Sparago A, Russo S, Cerrato F, et al. Mechanisms causing imprinting defects in familial Beckwith-Wiedemann syndrome with Wilms' tumour. Hum Mol Genet 2007; 16: 254-264.

11. Maas SM, Vansenne F, Kadouch DJ, et al. Phenotype, cancer risk, and surveillance in Beckwith-Wiedemann syndrome depending on molecular genetic subgroups. Am J Med Genet A 2016; 170: 2248-2260.

12. Mussa A, Peruzzi L, Chiesa N, et al. Nephrological findings and genotype-phenotype correlation in Beckwith-Wiedemann syndrome. Pediatr Nephrol 2012; 27: 397-406.

13. Kalish J, Deardorff M. Tumor screening in Beckwith-Wiedemann syndrome - To screen or not to screen? Am J Med Genet A 2016; 170: 2261-2264.
14. Bhatnagar S. Management of Wilms' tumor: NWTS vs SIOP. J Indian Assoc Pediatr Surg 2009; 14: 6-14.

15. Mussa A, Russo S, De Crescenzo A, et al. (Epi)genotype-phenotype correlations in Beckwith-Wiedemann syndrome. Eur J Hum Genet 2016; 24: 183-190.

16. Brioude F, Kalish J, Mussa A, et al. Expert consensus document: Clinical and molecular diagnosis, screening and management of Beckwith-Wiedemann syndrome: an international consensus statement. Nat Rev Endocrinol 2018; 14: 229-249.

17. Strenger V, Kerbl R, Dornbusch H, et al. Diagnostic and prognostic impact of urinary catecholamines in neuroblastoma patients. Pediatr Blood Cancer 2007; 48: 504-509.

18. Dome JS, Perlman EJ, Graf N. Risk Stratification for Wilms Tumor: Current Approach and Future Directions. Am Soc Clin Oncol Educ B 2014; 34: 215-223.

19. Kinoshita $Y$, Suminoe A, Inada $\mathrm{H}$, et al. The prognostic significance of blastemal predominant histology in initially resected Wilms' tumors: A report from the Study Group for Pediatric Solid Tumors in the Kyushu Area, Japan. J Pediatr Surg 2012; 47: 2205-2209.

20. McNeil DE, Langer JC, Choyke P, et al. Feasibility of partial nephrectomy for Wilms' tumor in children with Beckwith-Wiedemann syndrome who have been screened with abdominal ultrasonography. J Pediatr Surg 2002; 37: 57-60.

21. Al-Hussain T, Ali A, Akhtar M. Wilms Tumor. Adv Anat Pathol 2014; 21: 166-173.

22. Kliegman R, Lye PS, Bordini BJ, et al. Nelson pediatric symptom-based diagnosis. Elsevier, Philadelphia 2018; 816.

23. Munns CF, Batch JA. Hyperinsulinism and Beckwith-Wiedemann syndrome. Arch Dis Child Fetal Neonatal Ed 2001; 84: F67-69.

24. Niikawa N, Ishikiriyama S, Takahashi S, et al. The Wiedemann-Beckwith syndrome: pedigree studies on five families with evidence for autosomal dominant inheritance with variable expressivity. Am J Med Genet 1986; 24: 41-55. 